\title{
Mining Text Archives: Creating Readable Maps to Structure and Describe Document Collections
}

\author{
Andreas Rauber and Dieter Merkl \\ Institute of Software Technology, Vienna University of Technology \\ www.ifs.tuwien.ac.at/ andi www.ifs.tuwien.ac.at $/ \sim$ dieter
}

\begin{abstract}
With the ever-growing amount of unstructured textual data on the web, mining these text collections is of increasing importance for the understanding of document archives. Particularly the self-organizing map has shown to be very well suited for this task. However, the interpretation of the resulting document maps still requires a tremendous effort, especially as far as the analysis of the features learned and the characteristics of identified text clusters are concerned. In this paper we present the LabelSOM method which, based on the features learned by the map, automatically assigns a set of keywords to the units of the map to describe the concepts of the underlying text clusters, thus making the characteristics of the various topical areas on the map explicit.
\end{abstract}

\section{Introduction}

Mining unstructured text data requires the analysis of very high-dimensional data spaces, which makes it a challenging application arena for neural networks. In particular the self-organizing map (SOM) 2 as an unsupervised neural network model has been experiencing an increased popularity in a wide range of application arenas and is frequently used to produce a topologically ordered mapping of high-dimensional data spaces such as encountered in text document collections, cf. 1/35]. Among the many benefits we just refer to its stability and its ability to provide an intuitive visualization of high-dimensional data by producing a topology-preserving mapping from a high-dimensional input space to a usually two-dimensional map space.

However, interpreting the resulting trained maps is not as intuitive as it could be. This is in spite of the recent advance in sophisticated cluster visualization methods for the self-organizing map like the U-Matrix [8, Adaptive Coordinates or Cluster Connections techniques 4. While all of these methods focus on the visualization of cluster structure, it still remains a tedious task to interpret the mapping of the SOM as such, i.e. to analyze which attributes were relevant for a particular mapping to describe the characteristics of the clusters identified or, in the case of text mining, reveal the concepts of the various text document clusters. When we look at present applications of the SOM, we usually find it labeled manually, i.e. after inspection of the trained map a set of keywords is assigned 
to each unit or cluster to provide the user with some hints on the contents of the map. This process, obviously, is highly labour intensive by requiring manual inspection of the data mapped onto the units.

In this paper we present our LabelSOM method to automatically assign keywords to the units of a trained self-organizing map. We demonstrate the benefits of this method by using it to describe the topics of documents clustered by a self-organizing map. As a sample document archive we use the classsic TIME Magazine article collection consisting of articles from the TIME Magazine from the 1960's. The LabelSOM method allows to now automatically describe the categories of documents using the features learned by the SOM and thus assists the user in understanding the data collection presented by the map.

\section{The Self-Organizing Map and LabelSOM}

The $S O M L i b$ library [6] is based on the self-organizing map [2] (SOM), a popular unsupervised neural network model. The SOM abstracts from presented input data to provide a topology preserving mapping from a high-dimensional input space to a usually two-dimensional output space. It consists of a 2-dimensional grid of units with $n$-dimensional weight vectors. During the training process input signals are presented to the map in random order. An activation function is used to determine the winning unit as the unit showing the highest activation. Next, the weight vectors of the winner and its neighboring units are modified following some learning function to represent the input signal more closely. The result of this training process is a map providing a topology-preserving mapping in so far as similar input data are located close to each other on the 2-dimensional map display. The weight vectors, in turn, resemble as far as possible the input signals for which the respective unit serves as a winner.

With no a priori knowledge on the data, even obtaining information on the cluster boundaries as it is provided by a number of cluster boundary analysis methods for the SOM 4,8, does not reveal information on the relevance of single attributes for the clustering and classification process. In the LabelSOM approach we determine those vector elements (i.e. features of the input space) that are most relevant for the mapping of an input vector onto a specific unit. This is basically done by determining the contribution of every element in the vector towards the overall Euclidean distance between an input vector and the winners' weight vector, which forms the basis of the SOM training process.

The LabelSOM method is built upon the observation, that, after SOM training, the weight vector elements resemble as far as possible the corresponding input vector elements of all input signals that are mapped onto this particular unit as well as to some extent those of the input signals mapped onto neighboring units. Vector elements having about the same value within the set of input vectors mapped onto a certain unit describe the unit in so far as they denominate a common feature of all data signals of this unit. If a majority of input signals mapped onto a particular unit exhibit a highly similar input vector value for a particular feature, the corresponding weight vector value will be highly similar as 
well. We can thus select those weight vector elements, which show, by and large, the same vector element value for all input signals mapped onto a particular unit to serve as a descriptor for that very unit. This is done by calculating the so-called quantization error vector. It is computed for every unit $i$ as the accumulated distance between the weight vector elements of all input signals mapped onto unit $i$ and the unit's weight vector elements. More formally, this is done as follows: Let $C_{i}$ be the set of input patterns $x_{j}=<\xi_{i_{1}}, \ldots, \xi_{i_{n}}>\in \Re^{n}$ mapped onto unit $i$. Summing up the distances for each vector element $k$ over all the vectors $x_{j}\left(x_{j} \in C_{i}\right)$ and the corresponding weight vector $m_{i}=\left\langle\mu_{i_{1}}, \ldots, \mu_{i_{n}}\right\rangle$ yields a quantization error vector $q_{i}$ for every unit $i$ (Equation 11).

$$
q_{i_{k}}=\sum_{x_{j} \in C_{i}} \sqrt{\left(\mu_{i_{k}}-\xi_{j_{k}}\right)^{2}}, \quad k=1 . . n
$$

The quantization error for all individual features serves as a guide for their relevance as a class label. Selecting those weight vector elements that exhibit a corresponding quantization error of close to 0 thus results in a list of attributes that are shared by all input signals on the respective unit and thus describe the characteristics of the data on that unit. These attributes thus serve as candidate labels for regions of the map for data mining applications. Based on the ranking provided by the quantization error vector, we can decide to select either a set of labels exhibiting a quantization error vector value below a certain threshold $\tau_{1}$ as labels or simply choose a set of up to $n$ labels for every unit.

While this selection of labels may be used for standard data mining applications, in the text mining arena we are usually faced with a further restriction. Due to the high dimensionality of the vector space and the characteristics of the $t f \times i d f$ representation of the document feature vectors, we usually find a high number of input vector elements that have a value of 0 , i.e. there is a large number of terms that is not present in a group of documents. These terms obviously yield a quantization error value of 0 and would thus be chosen as labels for the units. Doing that would result in labeling the units with attributes that are not present in the data on the respective unit. While this may be useful for some data analysis tasks, where even the absence of an attribute is a distinctive characteristic, it is definitely not the goal in text mining applications where we want to describe the present features that are responsible for a certain clustering rather than describe a cluster via the features that are not present in its data. Hence, we need to determine those vector elements from each weight vector which, on the one hand, exhibit about the same value for all input signals mapped onto that specific unit as well as, on the other hand, have a high overall weight vector value indicating their importance. To achieve this we define a threshold $\tau_{2}$ in order to select only those attributes that, apart from having a very low quantization error, exhibit a corresponding weight vector value above $\tau_{2}$. In these terms, $\tau_{2}$ can be thought of indicating the minimum importance of an attribute with respect to the $t f \times i d f$ representation to be selected as a label. 

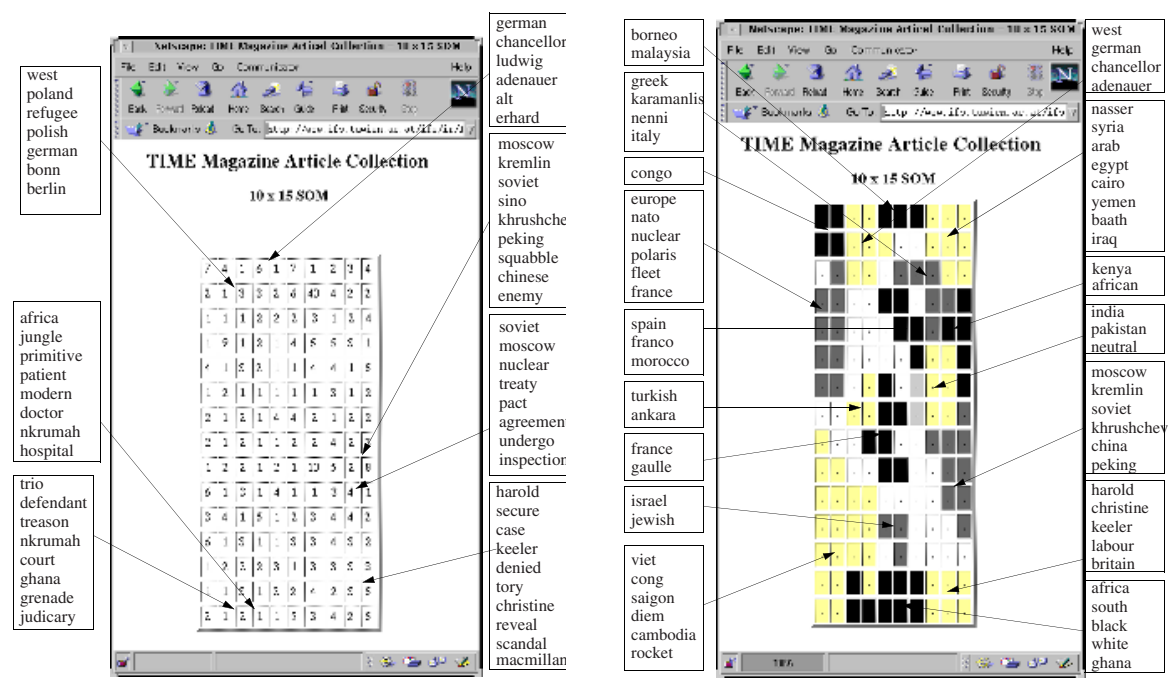

Fig. 1. a.) SOM with labels b.) Clusters identified by labels

\section{Experimental Results}

For the following experiments we use the TIME Magazine article collection. It consists of 420 articles from the TIME Magazine dating from the early 1960's.

To be used for SOM training, the articles are transformed into a vector representation following the vector space model of information retrieval. We use full-term indexing to extract a list of all words present in the document collection while applying some basic stemming rules. Furthermore, we eliminate words that appear in more than $90 \%$ or less than 3 articles, with this rule saving us from having to specify language or content specific stop word lists. The documents are thus are represented by a set of vectors using a $t f \times i d f$, i.e. term frequency times inverse document frequency, weighting scheme [7]. This weighting scheme assigns high values to terms that may be considered important in terms of describing the contents of a document and discriminating between the various articles. For the 420 TIME Magazine articles, the indexing process identified 5923 terms, thus describing the articles by 420 feature vectors of dimensionality 5923 .

These feature vectors are further used to train a self-organizing map consisting of $10 \times 15$ units. The resulting SOM is depicted in Figure 1 a where, due to space considerations, instead of listing the articles that are mapped onto a particular unit, we rather give the number of documents mapped onto the units. The SOM provides an ordered mapping of the articles in terms of the feature vector representation, meaning that articles exhibiting similar features and thus covering similar topics are located close to each other on the map. However, identifying which topics are covered in which part of the map requires the analysis of the individual articles. To be able to describe the clusters, we would now need to read the articles mapped onto the various units and then assign labels to the 
units in order to make the map readable, which even for document collections of this size is a very time-consuming task.

Figure 1 1 a also represents the result of applying the LabelSOM method to assign keywords to the individual units. Due to the limited space we can only present the labels for a subset of all units. However, the quality of the labels for the remaining units is similar 1 . The automatically created labels nicely describe the various documents that are mapped onto the respective units.

For example, the 4 articles mapped onto unit (9/10 $\sqrt{2}$ labeled soviet, moscow, nuclear, khrushchev, treaty, pact, agreement, undergo, inspection all deal with the talks taking place in Moscow under the supervision of Nikita Khrushchev concerning the ban of nuclear tests and the various conditions for an agreement like on-site inspections. Next to it, the 2 documents represented by unit $(9 / 9)$ labeled moscow, kremlin, soviet, stalin, peking, chinese, sino, enemy discuss the relationship between China and the Soviet Union. These units are part of a larger cluster dealing with the role of Moscow both in its relationship to the western world as well as to its neighbor China. While the labels for each unit serve as a detailed description of the articles on the particular unit, choosing only labels that are shared with neighboring units allows the creation of higher-level class labels. The units mentioned above, for example, are part of a larger cluster sharing labels like kremlin, moscow, khrushchev, soviet, china, chinese.

Cluster labels like these can be found in other regions of the map like e.g. in the upper left area of the map, where we have a cluster labeled west, germany and adenauer, for which the labels for the individual units reveal more detailed information by providing additional labels like chancellor, election, socialist, ludwig for unit (4/1) or poland, polish, refugee, bonn, berlin for unit (3/2).

To give some more examples, we find a rather large cluster labeled viet, cong, diem, rocket in the lower left corner of the map dealing with the political situation and the war in Vietnam, followed by a cluster reporting on africa with one subsection labeled ghana, nkrumah (3/15) dealing with the situation in Ghana under its dictator Kwame Nkrumah and the existence of Albert Schweizers primitive hospital under these conditions in the jungle of Ghana on unit (4/15) labeled nkrumah, primitive, patient, hospital, jungle. A second subsection of the Africa-cluster is labeled with, amongst others, south, africa, black, white, apartheit and deals with the apartheit policy in this area.

As a last example consider units $(11 / 10)$ and (12/10). From the labels it is obvious, that the corresponding documents deal with the British ProfumoKeeler scandal. The script behind this scandal is that British minister of defense John Profumo had an affair with Christine Keeler, who had connections to the Soviet secret service. From today's point of view it's kind of interesting how history repeats itself as in document T170 Profumo is quoted "There has been no impropriety between myself and Miss Keeler".

\footnotetext{
$\overline{1}$ The map is available for interactive exploration via our department webserver at http://www.ifs.tuwien.ac.at/ifs/research/ir/

${ }^{2}$ We will use the notation $(x / y)$ to refer to the unit located at column $x$ and row $y$.
} 
We can use the labels assigned to the various units to further identify clear cluster boundaries between topical clusters of documents. This can be achieved by identifying those labels of a unit that also appear as labels for one of the neighboring units, meaning that these units share some common features. Thus we can identify topical clusters by identifying areas in the map where the units have one or more labels in common. A representation of the resulting larger clusters with their corresponding labels is given in Figure 1 1 . Please note, that the granularity of the clusters identified depends on the number of labels assigned to every unit and the threshold $\tau_{2}$ for the minimum importance of a label in the $t f \times i d f$ representation. If fewer labels are assigned, we tend to identify a larger number of small clusters comprising only units covering highly similar topics. On the other hand, when we assign a larger number of labels to every unit, we can identify fewer, but larger topical clusters at a higher level of abstraction.

\section{Conclusions}

We presented the LabelSOM method, which provides a straightforward way for assigning labels to the units of a self-organizing map. Attributes that are shared by the input signals mapped onto a particular unit are used to describe that unit. This facilitates the interpretation and understanding of the contents of a SOM and the features that it learned, which is hardly possible without the additional information provided by the automatically created labels. Using the LabelSOM method largely improves the applicability of the SOM both in the field of data mining and data representation. The clusters identified by the map not only become intuitively visible as with the enhanced cluster visualization methods developed so far, but are also characterized, allowing the interpretation of the features the SOM has learned. Producing a labeled SOM of a text collection allows it to be read and understood in a way one would expect from manually indexed document archives.

\section{References}

1. S. Kaski, T. Honkela, K. Lagus, and T. Kohonen. WEBSOM-self-organizing maps of document collections. In Elsevir Publ. Elsevir Publications, 1997.

2. T. Kohonen. Self-Organizing Maps. Springer Verlag, Berlin, Germany, 1995.

3. D. Merkl. Text classification with self-organizing maps: Some lessons learned. Neurocomputing, 21(1-3), 1998.

4. D. Merkl and A. Rauber. Alternative ways for cluster visualization in self-organizing maps. In Proc. of the Workshop on Self-Organizing Maps (WSOM97), Helsinki, Finland, 1997.

5. A. Rauber and D. Merkl. Creating an order in distributed digital libraries by integrating independent self-organizing maps. In Proc. Int'l Conf. on Artificial Neural Networks (ICANN'98), Skövde, Sweden, 1998.

6. A. Rauber and D. Merkl. The SOMLib digital library system. In Proc. European Conference on Digital Library Systems, Paris, France, 1999. LNCS, Springer Verlag.

7. G. Salton. Automatic Text Processing: The Transformation, Analysis, and Retrieval of Information by Computer. Addison-Wesley, Reading, MA, 1989.

8. A. Ultsch. Self-organizing neural networks for visualization and classification. In Information and Classification. Concepts, Methods and Application, 1993. 